Journal of Universal Computer Science, vol. 26, no. 8 (2020), 904-928

submitted: 12/1/20, accepted: 13/7/20, appeared: 28/8/20 CC BY-ND 4.0

\title{
Exploring Virtual Reality in the Higher Education \\ Classroom: Using VR to Build Knowledge and Understanding
}

\author{
Gareth W. Young \\ (V-SENSE, Trinity College Dublin, Dublin, Ireland \\ YoungGa@TCD.ie) \\ Sam Stehle \\ (NCG, Maynooth University, Maynooth, Ireland \\ Sam.Stehle@MU.ie) \\ Burcin Yazgi Walsh \\ (NCG, Maynooth University, Maynooth, Ireland \\ Burcin.YazgiWalsh@MU.ie) \\ Egess Tiri \\ (NCG, Maynooth University, Maynooth, Ireland \\ Egess.Tiri.2017@MUmail.ie)
}

\begin{abstract}
Virtual reality (VR), as an informative medium, possesses the potential to engage students with immersive, interactive, and informative experiences. When presented in VR, immersive virtual environments (IVEs) can provide three-dimensional visual simulations that can be used to inform students about concepts in specific contexts that would be near impossible to achieve with more traditional teaching methodologies. It is proposed that existing learning frameworks can benefit from exploring the modalities of interaction that are presently afforded via VR from the experiential perspectives of the students. An evaluation is presented that focused on the appraisal of student experiences of immersive technologies as applied in a higher education context, specifically in the use of VR for the exploration of geomorphology theory by physical geography students. This research supports further development of the immersive learning discipline from three different perspectives. First, an empathy mapping method was applied to visualize student experiences and externalize our observed knowledge of student users for creating a shared understanding of their needs and to aid in lesson planning decision making when using VR in the classroom. Second, student experiences were captured using a technology-focused user experience questionnaire to obtain student attitudes immediately post-task. Finally, to assist teachers with the creation of a student-centered lesson plans that incorporate VR in the classroom, eight heuristic guidelines (focus, provocation, stimulation, collaboration, control, digital life, learner skills, multimodal experience) were developed. It is proposed that these findings can be used to provide support for the use of mixed reality and immersive virtual environments in learning that encompass the challenges faced by students and the interdisciplinary education community at large.
\end{abstract}

Key Words: Higher Education; Mixed Reality; Virtual Reality; User Experience Category: K.3, K.3.1, L.0.0, L.1.0, L.2.3, L.3.1, M.8 


\section{Introduction}

Contemporary mixed reality (MR) platforms can be used in multiple contexts to merge the real world with the virtual and vice-versa producing new environments where physical and digital objects can co-exist and be interacted with in real-time [Milgram et al. 1995]. In particular, the virtual reality (VR) industry is growing, with the market size of consumer hardware and software projected to increase from 6.2 billion U.S. dollars in 2019 to more than 16 billion U.S. dollars by 2022 , with unit shipments of VR devices expected to reach 12.5 million in 2020 [Statista 2020]. The effect of immersive virtual environments (IVEs) as pedagogical tools for primary and secondary education is a relatively well-examined area of MR research [Pellas et al. 2019, Freina and Ott 2015]. Nevertheless, with the recent proliferation and increased popularity in the use of low-cost, selfpowered head-mounted displays (HMD's) and smartphone technology, there has been relatively little analyses to explore their affordances in the higher education (HE) classroom and capture the experiential challenges that are being faced by students using immersive technology at this level [Wohlgenannt et al. 2019, Baxter and Hainey 2019].

In this formative study of VR applied in a HE classroom, we observe the experiential learning impacts of using VR as an immersive, interactive, and explorative platform for Physical Geography students. This research can be generalized to provide further insight into student experiences of explorative educational paradigms applied in the classroom that can also be more freely applied in other MR learning contexts. To achieve this, we visualized student attitudes and behaviors, assisting in the application of user-centered design (UCD) paradigms, and generated a set heuristics and guiding questions for assessing the learning potential of VR in the classroom. We focus on student experiences for the creation and application of educational VR materials; after all, the first principle of Wilfred J. Hansen's User Engineering Principles is to "KNOW THE USER" [Hansen 1971]. In the process of learning to "know" how students experience learning in commercial "off-the-shelf" IVEs, design processes that are sympathetic to this user group's needs can be better facilitated in future lesson plans and purpose-built educational software. The research presented in this manuscript was, therefore, structured to specifically focus upon the experiences of a community of users of which the agency for VR in HE will be focused and create heuristics as a high-level checklist for future software and lesson plan developments.

By constraining the presented study to a specific VR use-case context, this study facilitates future research and progressive design processes for HE software and MR lesson planning. This was to be achieved by building an understanding of the interests, attributes, and the needs of the primary users of this technology in HE - students - and builds empathy towards this user-group; a group that will 
benefit from learning via diverse MR platforms. In general, by building empathy and contributing to existing literature, future attempts to ensure educational immersive technologies applied in learning are acceptable to a larger proportion of users will be enabled. These insights will assist education orientated VR users by defining and understanding the actions of students within the much larger MR field as applied in a HE learning context. Moreover, as has been seen in other research, we hope to contribute to practices that apprise existing learning technology for the development of new systems on an iterative basis and assist in the refinement and improvement of VR research activities in this area [Sánchez et al. 2000, Chang et al. 2019].

Within this study, we aim to study student experiences of VR technology in explorative, knowledge-building exercises. In section 2, we investigate previous research in this area and create a strong foundation for our experiment methodology. In section 3, we present our experiment and outline the exact parameters and constraints of the study. In section 4 , we present our findings and discuss their meaning in a UCD context. In section 5, we present our heuristics for creating a UCD lesson plan and discuss the meaning and origins of each concerning our direct student observations. In section 6 , we acknowledge the limitations of our study and offer some suggestions for overcoming these issues. In our conclusions, we present the main takeaways and impact of our research.

\section{Background}

VR is "computer-generated environments that simulate the physical presence of people, objects, and realistic sensory experiences" [Freeman et al. 2017]. In theory, the advantages of training in VR simulations are that the IVE can facilitate users in the construction of a "context-dependent memory" [Norman 2017]. Therefore, by using an IVE in the classroom, it should be possible to take advantage of a student's memory of the real-world to stimulate new learning experiences and outcomes, such as is proposed in sense-making and knowledge-seeking theories [Dervin 1998]. This phenomenon has been observed in other educational contexts, such as music theory exercises [Timoney et al. 2018], gamified medical experiences [Vagg et al. 2016], and aircraft inspection training [Vora et al. 2002]. However, when multimedia educational materials are compared to real-world training, there have been mixed results [Sebrechts et al. 2003]; for example, in the transfer of skills in spatial navigation [Pollard eet al. 2020], medical simulations [Drews and Bakdash 2013], and rehabilitation [Levac et al. 2019]. Furthermore, early and indirect comparisons between real-world training, VR training, and no training, reported there to be no conclusive evidence to support the idea that knowledge transfer is better supported through the VR modality [Kozak et al. 1993]; however, more recent studies have indicated that train- 
ing in MR does not express a different outcome than training in nonsimulated, controlled environments and is equally effective at enhancing performance [Kaplan et al. 2020]. Moreover, it has been reported that regardless of the difficulties of using and managing new VEs, the technical, immersive, and social affordances of multi-user virtual environments (MUVEs), such as Second Life (an online virtual world developed by Linden Lab and launched in 2003), can offer numerous advantages in other learning contexts [Warburton 2009]. Historically, the use of VR in education has been well documented [Pantelidis 1993, Youngblut 1998, Freina and Ott 2015, Pellas et al. 2019], but early VR platforms were also regarded as a novelty that few students could realistically experience in a normative teaching space. With VR currently experiencing a resurgence, the barriers of VR for use in HE education are changing [Evans 2018b]. Discussing the use of newly accessible VR in the HE classroom is therefore arguably problematic without acknowledging and updating our awareness of previous work and exploring the role of contemporary VR in modern classrooms. Moreover, the topic is worthy of extended academic study, with the availability of new hardware and the various recommended educational uses for enhanced learning outcomes [Merchant et al. 2012, Song and Lee 2002, Yoo et al. 2018], learner satisfaction [Dicky 2011], virtual social interactions [Ke and Im 2013], and emotional value [Makransky and Lilleholt 2018].

\subsection{Exploratory Learning in Virtual Reality}

Conceptually, exploratory learning encourages a student to examine and investigate materials to discover relationships between existing knowledge and new data [Mavrikis et al. 2016]. This Constructionism learning theory was founded in Psychology studies that sought to explain how students acquire new knowledge and ultimately learn new skills [Papert 1986]. Fundamentally, constructivist approaches in education suggest that students can acquire knowledge through experience. As Fällman et al. stated "In order for the ideas of constructivist learning to succeed in education, we need to carefully examine and design assessment methods that make the deep but implicit knowledge students gain in constructivist learning conscious, visible and possible to assess" [Fällman et al. 1999]. In practice, students are led on field trips that present with many logistical challenges.

In an IVE, a student can be encouraged to explore in a constrained and orchestrated learning task or in a simulated situation that could not otherwise be realized; such as exploring remote glacial formations or the erosion of distant coastlines. Although the philosophies of explorative learning were not originally based on the use of MR technologies, the student can, however, benefit from the experience of immersive educational proficiencies that these technologies innately afford. Moreover, the factors of immersion [Curran 2018] that accompany 
existing VR practices serve only to further personalize the learning experience in a way that other educational technologies simply cannot.

\subsection{Immersive Virtual Environments in the Classroom}

As VR systems have tended in the past to have complex or unfamiliar user interfaces, they may potentially lose appeal with first-time or novice users. To further confound the use of VR in the classroom, not all students can enter an IVE through VR as a small number of users can experience adverse reactions when using head-mounted displays (HMDs); this includes nausea, dizziness, motion sickness, and headaches. However, this phenomenon, known as "simulation sickness", can be overcome with careful consideration of how a VR interaction is facilitated [Lewis-Evans 2018]. Still, VR is experiencing a renewal in commercial interest and is at present a flourishing technology that is increasingly being made available for everyday use [Bellini et al. 2016]. With the advent of new, faster, and more comfortable technology and the application of new VR design guidelines, many of these issues can be overcome; for example, see the many guidelines in place for VR developers from Oculus, Google, and InstaVR.

With the arrival of newer VR technologies, the restrictions on use have been considerably reduced. Furthermore, high-quality commercial software is openly available via online video game digital distribution platforms, such as Steam and the Oculus Store. While The HTC Vive, Valve Index, Oculus Rift, Oculus Quest, and PSVR all offer unique and advanced controllers for the manipulation of objects within the modern IVE, they exist in commercial environments that offer a more focused advancement of the field of VR in general [Evans 2018a]. Because of this, the potential of VR technology to be applied in the classroom compels revisiting, explicitly in the formative techniques applied in the evaluation of student experiences in explorative learning contexts. Therefore, the investigative methodologies presented in this manuscript are intended to explore student experiences of $\mathrm{VR}$, as is seen in other contemporary human-computer interaction (HCI) studies [Zelle and Figura 2004, Brown and Green 2016].

Specifically, the potential role of VR technology to facilitate Geography students in the exploration of classroom theories, such as uninhabitable or distant locations, as well as providing a platform for exploring real-world phenomena, such as river delta formations, are of explicit interest in an educational research context. To illustrate how IVEs can influence learning experiences of Geography, their use in educational contexts have been suggested for exploring locations that students could not easily access; observing real-world objects that could not be examined effectively without modifying time and space; interacting with real people in abstract or non-realistic ways [Stuart and Thomas 1991]. In more contemporary works, blended learning technologies have shown that digital learning 
environments can increase the level of academic achievements Geography students can achieve [Bondarenko, et al. 2019]. Moreover, when using Google Earth VR in the classroom, students have expressed greater learning satisfaction regarding the application of professional skills and have developed deeper cultural understandings of the phenomena they study [Hodgson et al. 2019].

Designing or enhancing a lesson plan with new technology is a difficult task, requiring the adaptation and creation of course content, context awareness, student attitudes, and, of course, getting to grips with the technology itself [Matuk et al. 2015]. Guidelines for best practices in teaching and learning are implemented to guide educators in providing safe and enjoyable immersive experiences for students when using VR equipment in HE [University of Toronto 2019]. By exploring and learning about student experiences, it is suggested that guidelines will be of some assistance for teachers and software designers when creating, implementing, and evaluating VR lesson plans; facilitating effective UCD approaches that support learner experiences with VR technology and IVEs. Heuristics in usability studies have been used since the early 90s', providing rules of thumb and not specific usability guidelines for software designers [Nielsen 1994, Shneiderman 2010]. By applying this structured model of analysis, it was possible to highlight specific areas of concern for students that could then be translated into guidelines and recommendations that inform future lesson plans and educational software design and support teachers in the application of a diverse range of learning theories and classroom methodologies.

Concerning the main goals of the current study, we aimed to collect user data that could be used to facilitate MR technology developers in the creation of IVEs in support of explorative learning in these types of roles. Education focused software is often created to increase a student's motivation and engagement with learning materials [Amory et al. 1998, Kearney 2004]. Furthermore, student user experience data will enhance the creation of clear lesson plans, providing systematic student-focused reflections for teachers to implement in practice via a heuristic checklist. Teachers face unique challenges in developing IVEs into lesson plans, which include compatibility with existing plans, technological integration, enabling multiple types of immersion, and maintaining immersive and non-immersive learning environments [Muñoz-Cristóbal et al. 2014]. The heuristics generated by this study sought to address some of these challenges for teachers by providing a student-centered assessment tool for new lessons and technology-driven interventions in the classroom. Moreover, we believe that education-focused software developers should be more contextually aware of the overall experiences of the students using their products or systems, especially in terms of how easy or pleasing they are to use beyond novelty.

Previous studies have demonstrated that VR materials have a varied effect on students in the classroom; therefore, we developed three main hypotheses to 
contribute new research findings to this field:

- H1. Students display varied previous experiences of VR and domain knowledge that must be considered when preparing to use VR in the HE classroom

- H2. Student experiences will be concerned with the attractiveness of VR, as well as the pragmatic and hedonic qualities of VR applied in the HE classroom that have various impacts upon lesson planning

- H3. The effects of student experiences observed in the use of VR in the HE classroom can be explored and applied to create a heuristic checklist to inform future UCD lesson-planning that includes the use of VR

\section{$3 \quad$ Experiment Methodology}

A total of 50 students from the Department of Geography were invited via email to attend a workshop on the topic of Geomorphology. This session was facilitated as part of a structured Geography module: Special Topics in Physical Geography - Principles of Geomorphology. A lesson plan for a student workshop from the previous semester was collaboratively augmented to integrate the use of Google Earth VR for exploring remote geomorphological phenomena in the classroom. The learning outcomes from an existing lesson plan were integrated into a new lesson that allowed students to explore geomorphological features in real-time. The lecturer was involved in creating the lesson plan and given technical and pedagogical support by the MR research team to prepare the activity based upon the predefined learning goals of the module. The experiment was conducted in the latter half of the second semester of the academic year, giving the students time to gather the necessary knowledge to build upon in an explorative context. The participants were sufficiently familiar with geomorphological concepts, having previously completed lectures on several interrelated topics within the field of geomorphology, and had had the opportunity to discuss and analyze the specific geomorphologic processes and theories in the classroom before the session. Furthermore, the students had already been assessed and had successfully passed the first semester's module assessment. After the invited responses were processed, participants were randomly divided into smaller project groups; each consisting of 3 members. The total participant pool consisted of 39 students, 17 males, and 22 females. The average age of the cohort was 21 years old $(S D=2.16)$. All members of the pool were educated to the European Qualifications Framework (EQF) level 4 or above and were currently enrolled in the second year of a Geography degree. To explore participant user types, the students were asked to identify their current domain knowledge and previous experiences of VR technology on a fully labeled 5-point Likert scale. As can be 


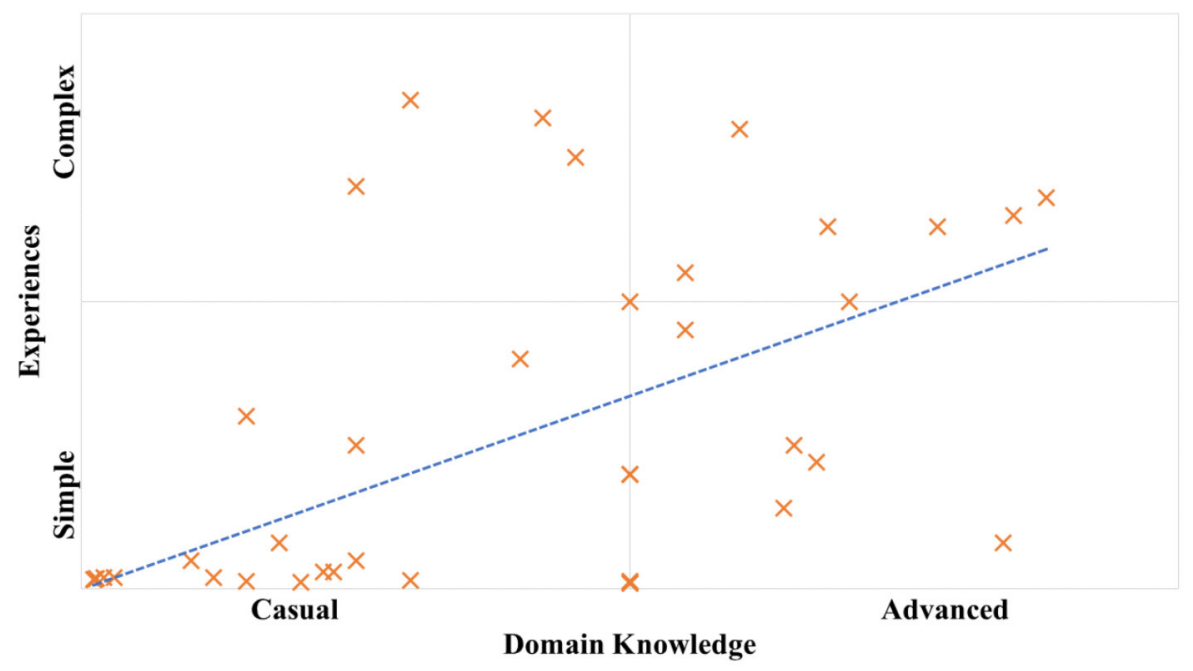

Figure 1: Student user-types - identifying the participants' domain knowledge of VR practices and previous experiences with VR technology (the dotted line represents the linear average).

seen in Figure 1, the participants presented with a relatively broad set of previous experiences and displayed an equally broad range of domain knowledge of VR technology.

To facilitate the session, a Dell Alienware Arura R6 (Intel i7-7700K Processor, dual NVIDIA GeForce GTX 1080 with 8GB GDDR5X each, and 64GB DDR4 memory) and HTC Vive (a 6DoF VR headset developed by HTC and Valve Corporation) were set-up in a familiar classroom environment, ensuring ecological continuity with the students' previous classroom experiences, see 2 for experiment setting. On the day of the group meetings, the participants were briefed on the session proceedings by the class lecturer (L; Figure 2), and the opportunity to ask any questions about VR was given; an MR researcher was available at this time to answer technical questions and was responsible for ensuring health and safety protocols, (R1; Figure 2). A student (S1VR; Figure 2) was then selected at random to enter Google Earth VR, a version of Google Earth in VR built specifically for Valve's Steam PC gaming platform that has been used in empirical research previously [Podwal 2016].

The starting location for all participants in VR was defined as a prefixed location max distance from Earth, over the Atlantic Ocean. The "Human Scale" option was toggled ON in Google Earth VR to enable a sense of measure for the geological formations visited. Students were then given a brief introduction to navigation by the MR researcher and allowed a short period to orientate them- 


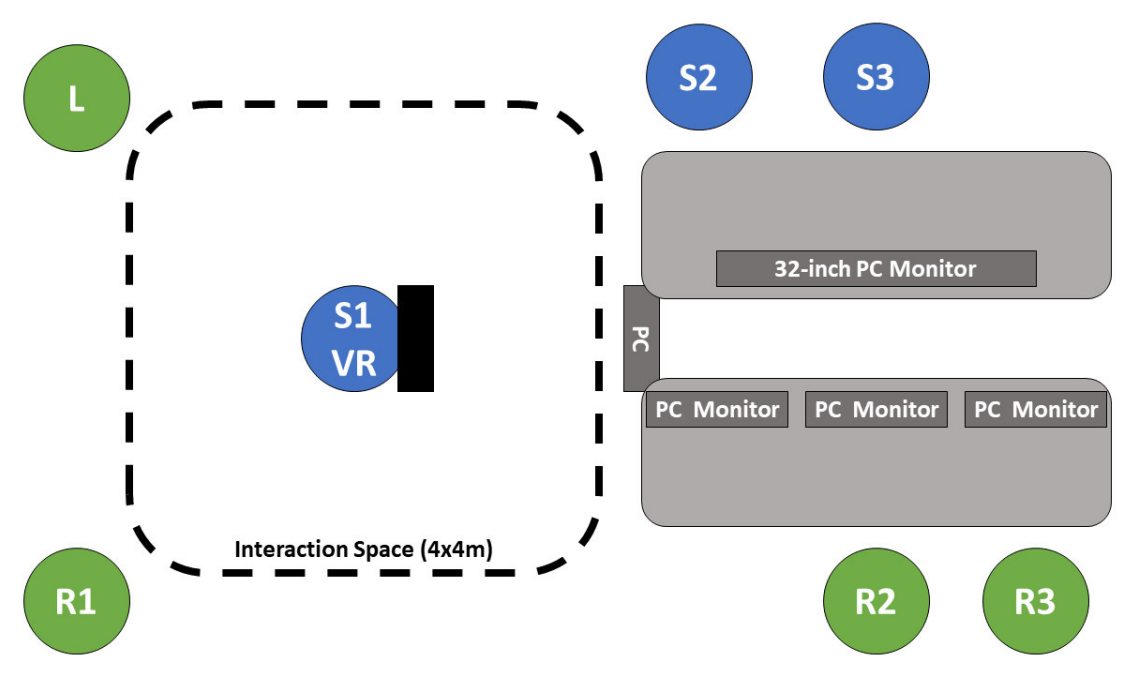

Figure 2: Experiment setting ( $\mathrm{L}=$ lecturer; $\mathrm{S} 1=$ student in VR; S2 \& S3 = students guiding S1; R1 = VR chaperone; R2 \& R3 = visible observation).

selves within the IVE and become familiar with the classroom materials (this time varied between students but did not exceed five minutes in all cases). The group outside of the IVE was given details on a randomly selected landmark location to guide the student in the IVE in the exploration of topographical formations. The lecturer then questioned the student in VR about the geomorphology of the given location, questioning that was constructed upon previous topics that had been discussed in the preceding lectures, such as the formation of sea stacks, glaciers, river deltas, and mountain ranges. The player position was reset at the end of the session, and the process was repeated for each student in the group. The total time on task was fixed at 10 minutes per student in VR and the overall session was to last one hour per group.

During each VR interaction, participant observations were collected by three MR and education research specialists to enable the development of a student empathy map, (R1, R2, R3; Figure 2. R1 collected direct Participant Observation data (the researcher is involved in the activity) while chaperoning $\mathrm{S} 1$. R2 \& R3 passively collected Non-participant Observation data (the researcher is separate from the activity) for S1, S2, and S3 during each session. The data consisted of information relating to Behavioral Observation (the researcher interprets people's behavior). In UCD, empathy maps help build understanding with end-users and the process of creating a map facilitates conversations and an aligned mental model between researchers [Gibbons 2018, Gray 2017, Bland 2016, Gray et al. 2010]. An empathy map consists of four separate focus 


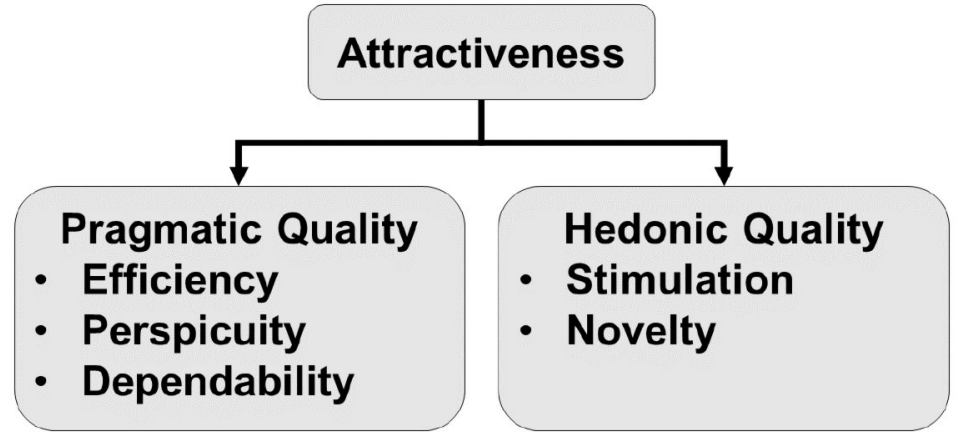

Figure 3: User Experience Questionnaire structure [Laugwitz et al. 2008].

areas that reflect observable behaviors undertaken during the task. These areas are focused on what the users 'Say', 'Feel', 'Think', and 'Do'. In addition to these main categories, two sub-categories representing the users' 'Pains' and 'Gains' are also used. The researchers then applied this data to create an aggregation of in-task student behaviors. The student empathy map was, therefore, to serve as an external visualization of the participant pool as they actively participated and completed the task.

Finally, during the post-task debrief stage of each session, the students were asked to complete a pen and paper version of the UEQ [Laugwitz et al. 2008]. The UEQ captured the students' immediate post-task impressions of the use of VR within the context of the classroom experience using 26 separate question items, each with a seven-stage semantic differential scale. Each scale of the UEQ ranged between -3 (extremely bad) and +3 (extremely good). Each scale is mapped to the overall attractiveness, perspicuity, efficiency, dependability, stimulation, and novelty of the interaction. The attractiveness rating scales of the UEQ served as a valence dimension for the goal-directed pragmatic qualities of perspicuity, efficiency, and dependability and as hedonic qualities of pleasant or unpleasant sensations regarding stimulation and novelty, see Figure 3 . It was then possible to combine the quantitative UEQ with the behavioral observational data to assess post-task how effective the workshop was on student experiences and how students behaved in the workshop.

\section{Results and Discussion}

\subsection{User Experience Questionnaire}

The UEQ results for attractiveness, pragmatic quality, and hedonistic quality aspects of student responses can be seen in Table 1 and Figure 4. These scales 


\begin{tabular}{lcccccc} 
Scale & Mean SD Confidence (Confidence Intervals) & $\boldsymbol{\alpha}^{*}$ \\
\hline Attractiveness & 2.21 & 1.01 & 0.32 & 1.89 & 2.53 & 0.94 \\
Perspicuity & 1.57 & 1.12 & 0.35 & 1.22 & 1.92 & 0.76 \\
Efficiency & 1.94 & 1.00 & 0.31 & 1.62 & 2.25 & 0.84 \\
Dependability & 1.54 & 0.72 & 0.23 & 1.31 & 1.76 & 0.15 \\
Stimulation & 2.25 & 0.90 & 0.28 & 1.97 & 2.53 & 0.87 \\
Novelty & 2.13 & 1.02 & 0.32 & 1.81 & 2.46 & 0.83
\end{tabular}

Table 1: Mean UEQ results with confidence intervals and Cronbach's AlphaCoefficient per scale $(\mathrm{n}=39)$. *Note: a reliability coefficient $(\alpha)$ of .70 or higher is considered acceptable in most scientific research conditions.

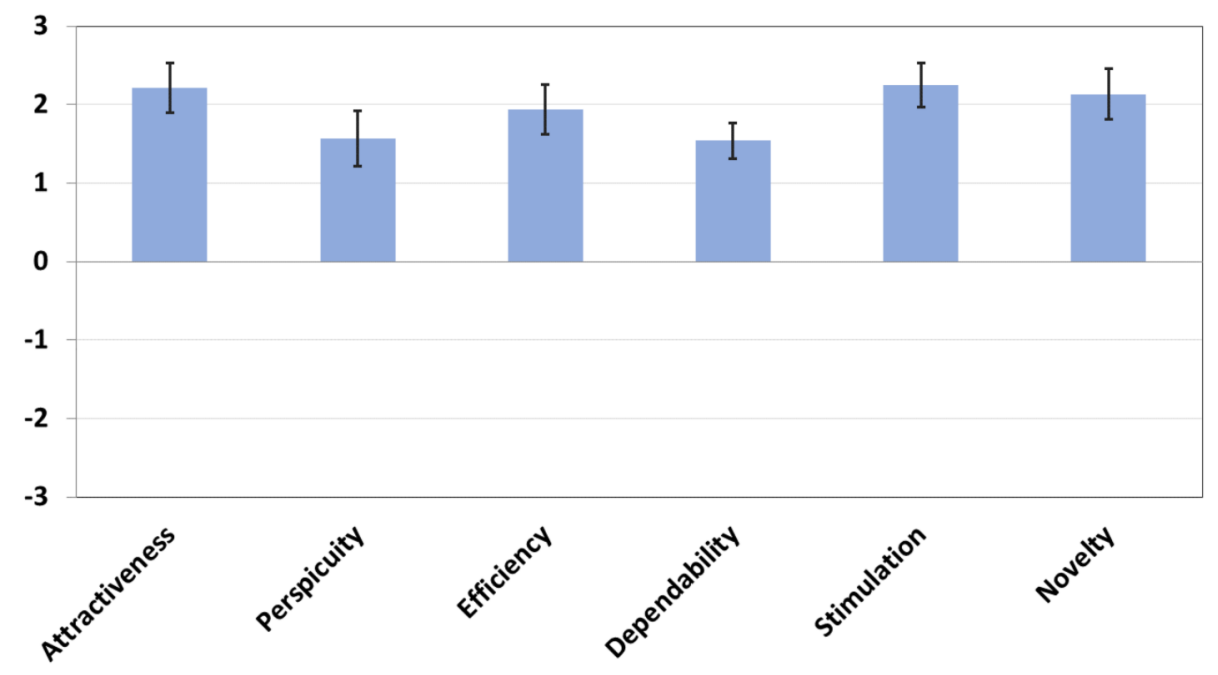

Figure 4: Mean values for student evaluations of VR with error bars representing the $95 \%$ confidence interval.

indicated how the pragmatic, use-quality elements performed as compared to the hedonistic, design-quality aspects of the experience. The pragmatic quality scales described the task-related quality rating and the hedonic quality defined other non-task related quality aspects.

The overall "attractiveness" of VR in the classroom was considered to be good $(M=2.21 ; S D=1.01)$. The pragmatic qualities of the interaction were all positively evaluated, indicating that the practicality and functionality of VR technology in this context, at a very basic level, could achieve its intended goals. Furthermore, because of the high pragmatic quality score, it can be stated that student satisfaction was achieved; meaning that when the students were using 
the IVE to realize their personal learning goals and that those goals were sufficiently achieved. Moreover, this score also indicated that the purpose of using VR technology in the classroom was clear and that the students understood how to use it effectively. The "efficiency" score $(M=1.94 ; S D=1.00)$ reflected the cognitive resources expended by the students concerning the accuracy and completeness of the goals they felt that they achieved [ISO 2010]. To support this, the students also rated "perspicuity" highly $(M=1.57 ; S D=1.12)$; meaning that the students thought it was easy for them to become familiar with the IVE while interacting with it through VR technology. This highlights that it was very easy for students to learn how to use the Google Earth VR user interface and navigation methodology. Finally, the student measure of "dependability" was rated highly $(M=1.54 ; S D=0.72)$. This further supports the idea that the students were in control, felt secure in task execution, and that the interaction response of the system in use was predictable throughout. However, this was not true for all students, as there was some measure of inconsistency in the AlphaCoefficient $(\alpha=0.15)$. The qualitative observations of the experiment serve to explore this occurrence further, as the students openly acknowledged that they had more difficulty than others using the headset, experienced some dizziness, and were limited to a single user at a time.

The evaluation of hedonic qualities also scored highly; meaning that the psychological and emotional experiences of the students were fulfilling. This score indicates that the students were enthused and that they enjoyed their learning experiences with VR technology. This inherently infers that the students' memories and previous knowledge-building experiences were evoked positively. The positive evocation of memory in this context signifies symbolic meanings in the learning experiences of the students' previous memories and their personal experiences during the task. The student ratings of "stimulation" support this $(M=2.25 ; S D=0.90)$, as the overall rating was positive. The stimulation dimension describes the extent to which VR technology supports the students' need for innovative and interesting functions, interactions, and stimulating content in the classroom. The students' positive evaluation of "novelty" $(M=2.13 ; S D=1.02)$ also represents how innovative the VR experience was perceived to be. Unfortunately, novelty ratings can, over time, become diminished and different sources of hedonic quality emerge, such as can be seen in the ritual application of PowerPoint presentations in the HE classroom. However, for the time being, the novelty factor of MR must be considered as an influential element of VR appraisal in the classroom.

\subsection{Empathy Mapping}

The observed actions of students during their VR sessions were emblematic of the self-reported experiences of the UEQ and several behaviors denoted confi- 


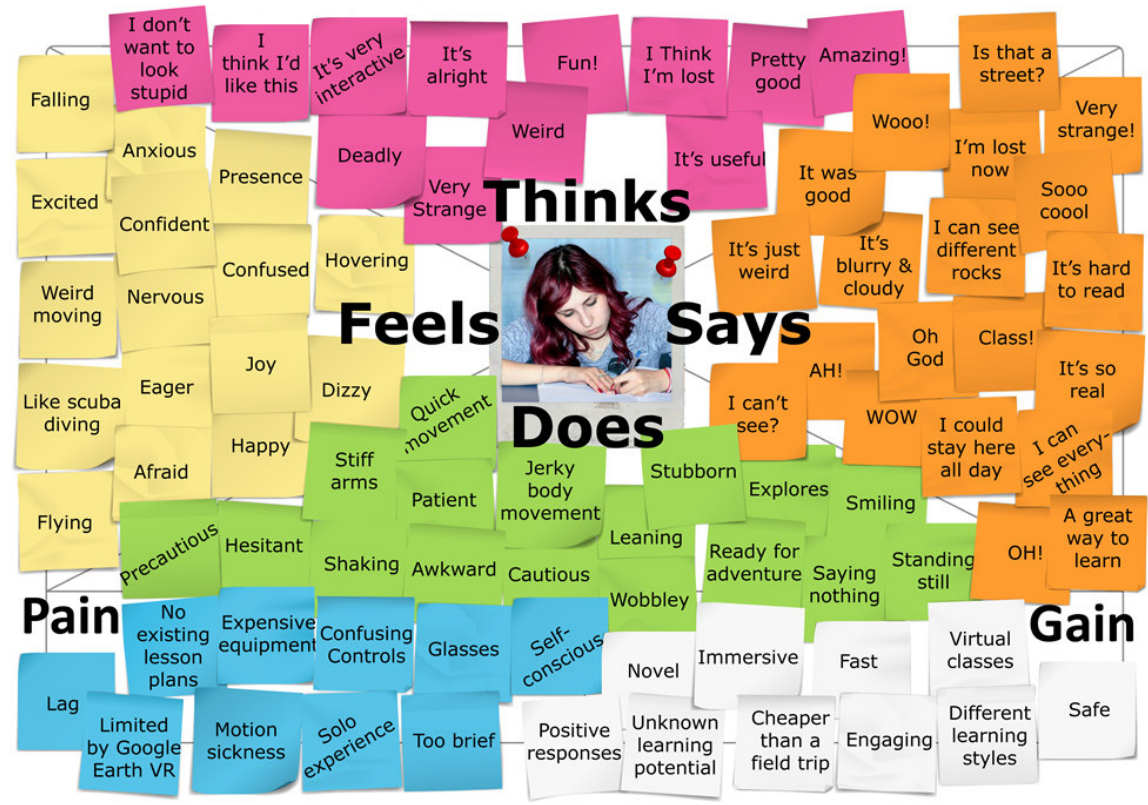

Figure 5: Student-focused empathy map.

dence and positive experiences (as expressed by the students mid-task as - feels: "confidence", does: "stubborn", pain: "limited by Google Earth VR"). Even for experienced VR users, of which a few took part in this study, the Google Earth VR package, the educational context of the activity, and the classroom setting introduced unfamiliar circumstances to their interactions. The resulting empathy map, therefore, consists of many expressions of surprise, confusion, and self-consciousness, but also positive evaluations of the overall educational experience. The visualization presented in Figure 5 therefore effectively communicates the qualitative side of what the students said, thought, did, and felt during the task; forming an understanding of their actions in-task and how they relate to their evaluation results in the post-task questionnaire. Although some confusion was expressed during the learning activity, the overall positive response concerning the stimulation of VR technology generally supports previous research comparing VR learning tools to other pedagogical learning tools.

As VR was reported as being an unfamiliar technology for some of the participants, see Figure 1, it can perhaps be considered as an overly complex sensory experience that students will eventually become more familiar and comfortable with over time. In previous research, when different levels of experience with VR technology are compared to the interestingness and motivation of educational VR 
experiences, an inverse relationship between previous experience and motivation was found [Virvou and Katsionis 2008]. This suggests that there is a potential for the complexity and unfamiliarity of VR to improve upon the learner's willingness to engage with technology in the classroom. Although some observations from the current study could likely be attributable to the few participants who identified as both experienced and knowledgeable, some participants also had low experience levels and still found the VR learning exercise to be stimulating and attractive above all other descriptions. Although, being limited by Google Earth VR could also be a consequence of experience with a full range of motion in VR gaming or intensely detailed and context-specific immersive training.

\section{Heuristics}

From student responses to the UEQ and our observations in the classroom, we have developed eight heuristic guidelines for teaching with VR. Our heuristics are each guided by a simple question for assessing whether the added value of the measure has been met by the classroom activity. Heuristic guidelines are intrinsic tools to these environments and are not intended to suggest a comparison to other teaching methods as an evaluative exercise. This has been performed for some hedonic qualities of VR elsewhere [Virvou and Katsionis 2008], but this is not a focus of our presented research. An overview of our effective UCD guidelines and heuristics can be seen in Table 2 .

\subsection{Focus}

Focus is a hedonic heuristic intended to measure the engagement of students with learning materials. The challenge with new learning media presents students with potentially discombobulating, confusing experiences, and unfamiliar environments and controls which can draw focus from the learning task. Of particular concern to VR is the impact of students feeling dizziness while using VR and the effect this has on student focus; which can be avoided with careful software design practices [Lewis-Evans 2018]. Potentially offsetting this effect is the novelty and encouragement of others using and learning through new means. Despite high ratings for the hedonistic qualities of VR from the UEQ of the experiment, empathy mapping suggested that focus can be impaired by the mechanics of the technology and the social situation it creates.

\subsection{Provocation}

A second hedonic quality related to innovative learning methods concerns the creative engagement of students with learning objectives. Learning design and course objectives influence the amount of creativity that students can express in 


\begin{tabular}{|c|c|c|c|}
\hline \multicolumn{2}{|c|}{ EQHeuristic } & Description & \multirow[b]{2}{*}{$\begin{array}{l}\text { Guiding Question } \\
\text { Does this technology fit in } \\
\text { with the current interests } \\
\text { of the students beyond the } \\
\text { novelty factor? }\end{array}$} \\
\hline \multirow{3}{*}{ 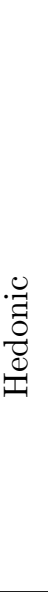 } & Focus & $\begin{array}{l}\text { Integrating VR technology } \\
\text { into education focused sce- } \\
\text { narios should help students } \\
\text { to stay engaged with learning } \\
\text { materials. }\end{array}$ & \\
\hline & Provocation & $\begin{array}{l}\text { VR devices and the learning } \\
\text { software applications they sup- } \\
\text { port should help students to } \\
\text { think imaginatively. }\end{array}$ & $\begin{array}{l}\text { Is the use of this technology } \\
\text { in the classroom innovative } \\
\text { and does it facilitate some } \\
\text { form of creativity? }\end{array}$ \\
\hline & Stimulation & $\begin{array}{l}\text { By using VR technology in the } \\
\text { classroom, the student should } \\
\text { be stimulated to become the } \\
\text { expert and to tutor others. }\end{array}$ & $\begin{array}{l}\text { Do students like or dislike } \\
\text { using this technology in the } \\
\text { classroom and do they en- } \\
\text { courage others to do so too? }\end{array}$ \\
\hline \multirow{5}{*}{ 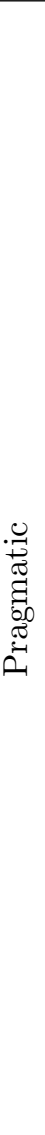 } & Collaboration & $\begin{array}{l}\text { VR technology should encour- } \\
\text { age students to explore col- } \\
\text { laboratively and enhance their } \\
\text { interactions with the learning } \\
\text { materials. }\end{array}$ & $\begin{array}{l}\text { Can students easily learn } \\
\text { how to use VR technology } \\
\text { and use it collaboratively in } \\
\text { the classroom? }\end{array}$ \\
\hline & Control & $\begin{array}{l}\text { VR technology should help stu- } \\
\text { dents feel a sense of responsi- } \\
\text { bility, improving their decision- } \\
\text { making skills, and supporting } \\
\text { existing knowledge. }\end{array}$ & $\begin{array}{l}\text { Does the student feel in con- } \\
\text { trol of the interaction and } \\
\text { confident that they are mak- } \\
\text { ing an informed and edu- } \\
\text { cated decision? }\end{array}$ \\
\hline & Digital Life & $\begin{array}{l}\text { Using VR technology in the } \\
\text { classroom should facilitate the } \\
\text { opportunity for students to de- } \\
\text { velop their digital citizenship } \\
\text { skills. }\end{array}$ & $\begin{array}{l}\text { Can students explore and } \\
\text { develop critical 21st-century } \\
\text { skills in VEs without unnec- } \\
\text { essary effort? }\end{array}$ \\
\hline & Learner Skills & $\begin{array}{l}\text { The introduction of VR tech. } \\
\text { into the classroom should pos- } \\
\text { itively enhance different learn- } \\
\text { ing experiences and create new } \\
\text { opportunities to learn. }\end{array}$ & $\begin{array}{l}\text { Is VR technology easy or dif- } \\
\text { ficult for students to become } \\
\text { familiar with? }\end{array}$ \\
\hline & $\begin{array}{l}\text { Multimodal } \\
\text { Experience }\end{array}$ & $\begin{array}{l}\text { By integrating VR technology } \\
\text { into the classroom students } \\
\text { should be connected to the } \\
\text { learning activity via all of their } \\
\text { senses. }\end{array}$ & $\begin{array}{l}\text { Is VR technology stimulat- } \\
\text { ing, exciting, and motivating } \\
\text { to use via multiple sensory } \\
\text { channels? }\end{array}$ \\
\hline
\end{tabular}

Table 2: Suggested effective heuristic guidelines to support learner experiences, categorized by Experiential Qualities (EQ). 
accomplishing their tasks. However, creative approaches to student provocation via teaching and learning through co-creation can be potentially be achieved. In this context, provocation attempts to measure the facilitation of VR technology for students' ability to exercise such creativity [Dawkins and Young 2020]. Novelty and provocation have a strong link, and what students feel and say can be attributed, in large part, to innovative methods. The novelty value was consistently rated by student respondents in the UEQ $(\alpha=0.76)$, see Table 1 , as well as in the observations, see Figure 5. Thus, guiding questions will evaluate provocation through the creativity students express in the pursuit of learning objectives.

\subsection{Stimulation}

Stimulation is a hedonic heuristic which coincides significantly with the stimulation measured through the user's experiences. As a heuristic for classroom learning, stimulation measures the willingness of students to take an active role in promoting the creative and effective use of VR to others and to use it for other learning experiences. Stimulation in this way is a behavioral measure of productive engagement and confidence in using the tool. Guiding questions are critical to evaluate stimulation, as this heuristic may not be observable in students while using MR. Therefore, the broader concepts of mixed reality could be approached to create multiple reality states, extending beyond virtual reality into augmented virtuality and augmented reality to provide a truly 'blended reality' learning environment [Peña-Ríos et al. 2012]. The empathy map presented in Figure 5 focused on the actions and expressions of the students while they were using VR as a knowledge-building tool, and so does not contain examples indicating the stimulation of users. However, during the experiment, students who were not immersed in the headset were observed volunteering assistance to the student in the IVE. No evaluative tools were available to test the motivation of helping; whether out of self-interest in completing a team-based assignment or out of genuine interest in facilitating the same positive experience they felt with the tool. Thus, stimulation is measured through questions that engage with students' passing their positive experiences in the classroom to other students and other learning contexts.

\subsection{Collaboration}

Collaboration is the first pragmatic heuristic quality which should be considered when using VR as a learning tool. Collaboration among users tries to address one of the "pains" expressed by students in our experiment; that VR experiences are largely solo experiences. The learning outcomes and lesson design incorporated teamwork-based objectives which required participation from members of 
the student group who were not using the headset, although those interactions were far less significant for the learning activity than the immersive experience. Collaborative learning increases the efficiency of the activity through the involvement of more than a single student, particularly for the individuality of being immersed in VR; although the efficiency of the learning activity was rated highly by most students $(M=1.94 ; S D=1.00 ; \alpha=0.84)$. To support this finding, VR has also been explored as a tool within higher education for collaborative work among team members working together in an IVE, but in remote locations from one another [Oprean et al. 2017, Peña-Ríos et al. 2012]. Although the students acknowledged the largely solo experience, they also expressed their thoughts on the potential for using the platforms for collaboration through such activities as "virtual classes" and field trip opportunities. Collaboration encourages new ways of thinking about and using the technology and guiding questions should consider the added value of using the immersive experience as a tool to work and learn along with others inside and outside of VR environments, as well as remotely.

\subsection{Control}

The pragmatic quality of control measures the ability of students to affect their ability to learn through the responsiveness of the tool to their inputs. Having control enables students to take responsibility for their learning through interactivity and the application of knowledge to the learning activity. Student responses to the user experience questionnaire indicated some concern about the control that is offered by the VR experience by rating the dependability of the activity slightly lower on average than all other hedonic or pragmatic qualities as well as a very low $\alpha(M=1.54 ; S D=0.72 ; \alpha=0.15)$. Dependability and control are linked through the responsiveness of the tool to the actions taken by the student. This low dependability ranking may also refer to the individual experience offered by the VR headset, where the group member using the tool was the only one able to shift the viewing perspective of the geological environment. Some students noted experiences out of their control during the experience, including "motion sickness", the activity being "too brief", and other technical expectations not being met resulting in "lag" and limits imposed by the Google Earth VR medium. Guiding questions should then emphasize the confidence that users have in the decisions that they make and the responsiveness to their input in pursuit of learning.

\subsection{Digital Life}

VR is a modern digital tool that presents the chance for students to engage with digital life and citizenship. Although digital life is a democratizing force 
[Mossberger et al. 2007], it also introduces fear for the ways that young digital citizens interact and interpret interpersonal digital spaces [LeBlanc 2018]. Given the novelty of MR technology and its growing implications in a twenty-firstcentury digital society, learning activities that utilize VR technology give many students their first exposure to an IVE, and therefore have a responsibility to also encourage students to engage with broader societal issues and meanings around digital representations and interactions. The controlled environment of the classroom and the lesson plan provided an ideal situation for introducing students to ways of critically thinking about and using new technologies. Evaluative questions should explore the student VR users' ability to translate their experiences into broader skills applicable to digital social spaces and without unnecessary effort. Considering the effects on digital citizenship via learning through VR experiences brings students from a position of learning about MR tools to learning with them.

\subsection{Learner Skills}

As a constructivist learning tool, the experiential context of learning in IVEs is an important way to both use and improve learner skills beyond memorization. Learner skills are potentially an issue when learning is done through an unfamiliar medium such as VR. Although students reported having a range of domain knowledge, their experience with VR in practice was still somewhat simple, rather than complex, see Figure 1, suggesting that they have not had many VR experiences in either the context of learning or playing. Those students who find VR experiences to be familiar may be rare to begin with, however, as indicated by the highly varied responses to the question of perspicuity, the ease at which learners can become familiar with the tool is a positive potential outcome for MR learning environments. The "unknown learning potential" of the platform was expressed as a positive aspect of the tool - a "gain" - which indicated a willingness to learn from the unfamiliar. Without previous experience using VR technology, perspicuity was expected to be lower on average. Many of the observed behaviors recorded in the empathy map of Figure 5 indicated the unfamiliarity that students have with the technology. However, unfamiliarity did not detract from the self-reported stimulation of students, so VR applications may as easily stimulate new styles of learning as they do allow certain types of learners to thrive. Evaluative questions may thus consider the novel ways that VR motivates students to learn new material and the ease at which familiarity and the development of learning skills can be achieved.

\subsection{Multimodality}

The VR classroom experience is unlike any offered before when it comes to merging learner experiences around a single multimodal tool. Visual, auditory, haptic, 
imaginative, and interactive, VR engages students, stimulating them in different ways and the creative medium allows also for exploring new multimodal experiences of learning in combinations they may not have had the opportunity to explore before. In previous studies, the multisensory experience of VR has helped students with learning disabilities which hamper traditional classroom evaluations, such as dyslexia, perform to the same standard of learning assessment as non-dyslexic classmates [Kalyvioti and Mikropoulos 2013]. Furthermore, audiotactile feedback has been shown to impact positively upon qualitative factors in creative practice [Young et al. 2017]. Students expressed new sensations which allowed them to experience learning with all senses engaged: "flying", "falling", "scuba diving", "I can see everything". Importantly, these unique experiences merging multiple sensory experiences coincide with the learning activity, such that it is motivating to use new methods of stimulation, and such methods allow students to explore concepts in ways they otherwise would not have the opportunity to. Guiding questions would consider the ways that multimodality leads to new insights and understandings for students in the context of specific subjects.

\section{Observed Constraints of Using VR in the HE Classroom}

To structure and explore the impact of VR in this applied HE context, this study assessed the effects of an IVE VR system on the directly observed user experiences of students in the HE classroom. The heuristics we have created are intended to allay or highlight some of the observed constraints or 'pains' of using VR in the classroom; however, we recognize that not all of the student issues we have observed can be easily mitigated. Some points that are worthy of particular note are the more obvious constraints of using VR in the classroom; such as the physical difficulties of using VR technology that presented while observing student behavior. Although all the students were physically able to complete the task, light motion sickness, and hesitance in using new technology were also noted. Furthermore, several students were impeded by wearing glasses. Additionally, some students also had personal difficulties in interacting with the other students when exploring the IVE and observing the topographical formations due to the large size of the VR headset in relation to the individual student. These types of observations show how the parameters of the experiment task and physicality interact with the overall learning experience.

Further to this and considering the lack of experience some of the participants displayed, the students expressed little in the way of questioning the role of VR technology or how to interact with it effectively. The pressure from peers, unfamiliar observers, and the presence of the course lecturer likely led to less willingness to ask for help; although many individuals would volunteer assistance without asking. The lack of questioning from the participants is perhaps 
indicative of their willingness to explore the virtual environment by themselves, regardless of the expressed limitations in using VR technology, and to engage with the topic of geomorphology within the limited constraints established by the task. The virtual reality medium and this study's experimental design successfully demonstrated the constructivist pedagogy of learning through experience and exploration [Sánchez et al. 2000, Papert 1986]. Students embraced this form of experiential learning, see Figures 4 and 5, as seen through their expressions of gains, which revolved around the teaching potential of VR.

In the presented manuscript, we have observed and measured students' experiences of using VR in the HE classroom, which has value and contributes to the field in and of itself, with room to garner further depth of knowledge in future studies. The experiment presents formative data to create and introduce a high-level heuristic, but further contextualized testing of this tool is required to validate its construction and use in practice. Future work will involve testing this tool with teachers to gain insight into their perspectives on lesson planning and achieving learning objectives with VR technology. Furthermore, while student experiences of VR in a collaborative learning task/environment were successfully evaluated, the analysis of task and learning activity complexity was outside of the scope of the presented study. Moreover, as VR is currently being used as a new teaching tool, it innately involves a novelty factor that presents as an interactive effector component in the study of user experiences with emergent technologies. Therefore, longitudinal research is also required to include the study of VR over multiple semesters or years as well as multiple discipline-specific analysis scenarios to validate their effective use.

\section{Conclusions}

Previous research has highlighted that teachers should seek out assistance from colleagues when redesigning their curricula, particularly when focusing on learning goals, and they should also perhaps consider making MR a more regular occurrence in the classroom [Patterson and Han 2019]. A virtual field experience platform inventory has been created to provide guiding questions for teacher educators to use when selecting and using a virtual field trip platform. To expand these theories beyond teachers, this manuscript presents an evaluation that focused on the appraisal of student experiences of IVEs as applied in a HE context; specifically, in the use of VR technology for the geospatial exploration of physical geography principles. The primary aim of the evaluation was to measure student responses to using VR technology in the classroom and reveal the experiential conditions that arise from such applications. Fundamentally, the students expressed an overall positive response to the attractiveness, hedonistic, and pragmatic effects of VR applied in this context. Furthermore, we successfully 
measured the students' experiences and attitudes towards the use of VR technology in the classroom by making it possible to explore and examine inaccessible places and topographical formations in the classroom. Through this foundational classroom use-case, an IVE presented via VR technology successfully facilitated geography students in experiencing real-world environments which would be difficult, expensive, and unsafe to visit in real life. This also supports the more general arguments in which "VR allows students to visualize abstract concepts, to take part in and interact with events that for reasons of distance, time, scale, safety, or money would not otherwise be conceivable" [Fällman et al. 1999]. Student experiences were measured, and their behaviors were observed during their classroom experience, to explore the value of VR in this context. The results of the analysis provided useful findings in relating the scope of VR in knowledge building exercises.

The UEQ examined student responses in six areas; attractiveness, perspicuity, efficiency, dependability, stimulation, and novelty. Student responses among these measures were overwhelmingly positive, with only small variations in mean response score and Alpha-Coefficient $(\alpha)$. These specific areas responded to by students indicated that classroom VR tools are attractive, motivating, and practical tools for learning in higher education. Moreover, observations of the students while using VR during this experiment revealed attitudes and expressions which provide further context to the ways that students interact with the technology. These observations were then further separated into 6 categories of "thinks", "feels", "says", "does", "pains", and "gains" for empathy mapping. These specific expressions are perhaps typical of first-time users of new technology, but also show that students successfully assessed the tool for suitability in the classroom. This study provides some assurance that students do not solely engage with virtual environments for play, but they can be used productively in many other ways.

We used the empathy mappings to identify areas of further growth for MR technology in the classroom. Bringing together student responses from the questionnaire, broader observations of student VR users from the literature, and previous research, this project has generated eight UCD heuristic guidelines for effective lesson planning and educational software development for use in the HE classroom. Consisting of both hedonic and pragmatic factors, these heuristics were directly based on our students' experiences. Related guiding questions are necessary to ask of students and the methodology of the VR learning activity. As an unfamiliar tool in student learning, the heuristics proposed here will guide the implementation of virtual experiences in the classroom. Further research, building on the eight roles that VR can play in an educational context [Stuart and Thomas 1991], will utilize these heuristics to create a more effective UCD process that will leverage the full potential of MR experiences in HE. 


\section{Acknowledgments}

This publication has emanated from research conducted with the financial support of the Science Foundation Ireland (SFI) under the Investigators' Award Program for the Building City Dashboards Project at Maynooth University (15/IA/3090) and the V-SENSE Project at Trinity College Dublin (15/RP/2776). The authors acknowledge the contribution of Martha Coleman from the Department of Geography at Maynooth University University for facilitating this research.

\section{References}

[Amory et al. 1998] Amory, A., Naicker, K., Vincent, J., Adams, C.: "Computer games as a learning resource"; Proc. EdMedia+ Innovate Learning, AACE (Association for the Advancement of Computing in Education), Waynesville (1998), 50 - 55.

[Baxter and Hainey 2019] Baxter, G., Hainey, T.: "Student perceptions of virtual reality use in higher education"; Journal of Applied Research in Higher Education, 12,3 (2019), 413 - 424.

[Bellini et al. 2016] Bellini, H., Chen, W., Sugiyama, M., Shin, M., Alam, M., Takayama, D.: "Virtual Augmented Reality: Understanding the race for the next computing platform"; Goldman Sachs Group, New York City (2016).

[Bland 2016] Bland, D.: "Agile coaching tip - What is an empathy map?"; (Accenture) Retrieved April 24, 2020, from https://www.solutionsiq.com/resource/blogpost/what-is-an-empathy-map/ (2016).

[Bondarenko, et al. 2019] Bondarenko, O., Mantulenko, S., Pikilnyak, A.: "Google classroom as a tool of support of blended learning for geography students"; arXiv preprint arXiv:1902.00775 (2019).

[Brown and Green 2016] Brown, A., Green, T.: "Virtual reality: Low-cost tools and resources for the classroom"; TechTrends, 60, 5 (2016), $517-519$.

[Chang et al. 2019] Chang, S. C., Hsu, T. C., Kuo, W. C., Jong, M. S. Y.: "Effects of applying a VR-based two-tier test strategy to promote elementary students' learning performance in a Geology class"; British Journal of Educational Technology, 51, 1 (2019), $148-165$.

[Curran 2018] Curran, N.: "Factors of Immersion"; The Wiley Handbook of HumanComputer Interaction. Wiley, Hoboken (2018), 239 - 254.

[Dawkins and Young 2020] Dawkins, O., Young, G. W.: "Ground Truthing and Virtual Field Trips"; Immersive Learning Research Network Workshop (2020).

[Dervin 1998] Dervin, B.: "Sense-making theory and practice: an overview of user interests in knowledge seeking and use"; Journal of knowledge management, 2, 2 (1998), 36 - 46.

[Dicky 2011] Dickey, M. D.: "The pragmatics of virtual worlds for K-12 educators: Investigating the affordances and constraints of active worlds and second life with K-12 in-service teachers"; Educational Technology Research and Development, 59, 1 (2011), 1 - 20.

[Drews and Bakdash 2013] Drews, F. A., Bakdash, J. Z.: "Simulation training in health care"; Reviews of Human Factors and Ergonomics, 8, 1 (2013), 191 - 234.

[Evans 2018a] Evans, L.: "The re-emergence of Virtual Reality"; Abingdon: Routledge (2018).

[Evans 2018b] Evans, L.: "Barriers to VR use in HE"; The VR/AR Conference 2018, 18 (2018), 3 - 13. 
[Fällman et al. 1999] Fällman, D., Backman, A., Holmlund, K.: "VR in Education: An Introduction to Multisensory Constructivist Learning Environments"; Proc. Conference on University Pedagogy, Sweden (1999).

[Freeman et al. 2017] Freeman, A., Adams Becker, S., Cummins, M., Davis, A., Hall Giesinger, C.: "NMC/CoSN Horizon Report: 2017 K-12 Edition"; Austin: The New Media Consortium (2017).

[Freina and Ott 2015] Freina, L., Ott, M.: "A literature review on immersive virtual reality in education: state of the art and perspectives"; Proc. The International Scientific Conference eLearning and Software for Education 1, 133 (2015), 10 - 1007.

[Gibbons 2018] Gibbons, S.: "Empathy Mapping: The First Step in Design Thinking"; Retrieved January 15, 2018, from https://www.nngroup.com/articles/empathymapping/ (2018).

[Gray 2017] Gray, D.: "Updated Empathy Map Canvas"; (Medium) Retrieved April 24, 2020, from https://medium.com/the-xplane-collection/updated-empathy-mapcanvas-46df22df3c8a (2017).

[Gray et al. 2010] Gray D, Brown S, Macanufo J.: "Gamestorming: A playbook for innovators, rulebreakers, and changemakers"; Sebastopol: O'Reilly Media, Inc. (2010).

[Hansen 1971] Hansen, W. J.: "User Engineering Principles for Interactive Systems"; Proc. The Fall Joint Computer Conference, Atlantic City (1971).

[Hodgson et al. 2019] Hodgson, P., Lee, V. W., Chan, J. C., Fong, A., Tang, C. S., Chan, L., Wong, C.: "Immersive virtual reality (IVR) in higher education: Development and implementation"; in Tom Dieck M., Jung T. (eds) Augmented Reality and Virtual Reality, Springer, Cham (2019), 161-173.

[ISO 2010] ISO (International Organization for Standardization): "ISO 9241-210:2010 (en): Ergonomics of human-system interaction. Part 210: Human-centered design for interactive systems (ISO/TC 159/SC 4)"; Retrieved June 30, 2017, from https://www.iso.org/obp/ui/iso:std:iso:9241:-210:ed-1:v1:en (2010).

[Kalyvioti and Mikropoulos 2013] Kalyvioti, K., Mikropoulos, T. A.: "A virtual reality test for the identification of memory strengths of dyslexic students in higher education"; J. UCS (Journal for Universal Computer Science), 19, 18 (2013) 2698 2721.

[Ke and Im 2013] Ke, F., Im, T.: "Virtual-reality-based social interaction training for children with high-functioning autism"; The Journal of Educational Research, 106, 6 (2013), $441-461$.

[Kearney 2004] Kearney, P.: "Engaging young minds-Using computer game programming to enhance learning"; Proc. EdMedia: World Conference on Educational Media and Technology, Association for the Advancement of Computing in Education (AACE), Lugano (2004), 3915 - 3920.

[Kaplan et al. 2020] Kaplan, A. D., Cruit, J., Endsley, M., Beers, S. M., Sawyer, B. D., Hancock, P. A.: "The Effects of Virtual Reality, Augmented Reality, and Mixed Reality as Training Enhancement Methods: A Meta-Analysis"; Human Factors (2020).

[Kozak et al. 1993] Kozak, J. J., Hancock, P. A., Arthur, E. J., Chrysler, S. T.: "Transfer of training from virtual reality"; Ergonomics, 36, 7 (1993), 777 - 784.

[Laugwitz et al. 2008] Laugwitz, B., Held, T., Schrepp, M.: "Construction and evaluation of a user experience questionnaire"; In Symposium of the Austrian HCI and Usability Engineering Group, Berlin: Springer (2008), 63 - 76.

[Levac et al. 2019] Levac, D. E., Huber, M. E., Sternad, D.: "Learning and transfer of complex motor skills in virtual reality: a perspective review"; Journal of NeuroEngineering and Rehabilitation, 16, 1 (2019), 1 - 15.

[LeBlanc 2018] LeBlanc, M., Furlong, C., Leger, M. T., Freiman, V.: "Digital Citizenship in a Global Context: The Relationships between Young People and the Digital World, the Actions they take and the Issues Associated with those Actions"; Proc. Society for Information Technology, Teacher Education International Conference, Washington: Association for the Advancement of Computing in Education (AACE) (2018), 363 - 371 
[Lewis-Evans 2018] Lewis-Evans, B.: "A Short Guide to User Testing for Simulation Sickness in Virtual Reality"; In A. Drachen, P. Mirza-Babaei, L. Nacke (Eds.), Games User research. Oxford: Oxford University Press (2018), 501 - 506.

[Makransky and Lilleholt 2018] Makransky G, Lilleholt L.: "A structural equation modeling investigation of the emotional value of immersive virtual reality in education"; Educational Technology Research and Development. 66, 5 (2018), 1141 1164.

[Matuk et al. 2015] Matuk, C. F., Linn, M. C., Eylon, B. S.: "Technology to support teachers using evidence from student work to customize technology-enhanced inquiry units"; Instructional Science, 43, 2 (2015), 229 - 257.

[Mavrikis et al. 2016] Mavrikis, M., Gutierrez-Santos, S., Poulovassilis, A.: "Design and evaluation of teacher assistance tools for exploratory learning environments"; International Conference on Learning Analytics Knowledge, Edinburgh (2016), 168 -172 .

[Merchant et al. 2012] Merchant, Z., Goetz, E., Keeney-Kennicutt, W., Kwok, O., Cifuentes, L., Davis, T.: "The learner characteristics, features of desktop 3D virtual reality environments, and college chemistry instruction: A structural equation modeling analysis"; Computers Education, 59, 2 (2012), 551 - 568.

[Milgram et al. 1995] Milgram, P., Takemura, H., Utsumi, A., Kishino, F.: "Augmented reality: A class of displays on the reality-virtuality continuum"; In International Society for Optics and Photonics: Telemanipulator and telepresence technologies, 2351 (1995), $282-292$.

[Mossberger et al. 2007] Mossberger, K., Tolbert, C. J., McNeal, R. S.: "Digital Citizenship: The Internet, society, and participation (1 ed.). Massachusetts: MIT Press (2007).

[Muñoz-Cristóbal et al. 2014] Muñoz-Cristóbal, J. A., Martínez-Monés, A., AsensioPérez, J. I., Villagrá-Sobrino, S., Hoyos-Torio, J. E., Dimitriadis, Y. A.: "City Ads: Embedding Virtual Worlds and Augmented Reality in Everyday Educational Practice"; J. UCS (Journal for Universal Computer Science), 20, 12 (2014), 1670 - 1689.

[Nielsen 1994] Nielsen, J.: "Heuristic evaluation"; In Nielsen, J., and Mack, R.L. (Eds.) Usability Inspection Methods; New York: John Wiley \& Sons (1994).

[Norman 2017] Norman, K. L.: "Cyberpsychology: An introduction to humancomputer interaction"; Cambridge: Cambridge University Press (2017).

[Oprean et al. 2017] Oprean, D., Simpson, M., Klippel, A.: "Collaborating remotely: an evaluation of immersive capabilities on spatial experiences and team membership"; International Journal of Digital Earth, 11 (2017), 420 - 436.

[Pantelidis 1993] Pantelidis, V.: "Virtual reality in the classroom"; Educational Technology, 33, 4 (1993), 23 - 7.

[Papert 1986] Papert, S.: "Constructionism: A new opportunity for elementary science education"; Cambridge: MIT Media Laboratory, Epistemology and Learning Group (1986).

[Patterson and Han 2019] Patterson, T., Han, I.: "Learning to Teach with Virtual Reality: Lessons from One Elementary Teacher"; TechTrends, 63, 4 (2019), 463 - 469.

[Pellas et al. 2019] Pellas, N., Kazanidis, I., Palaigeorgiou, G.: "A systematic literature review of mixed reality environments in K-12 education"; Education and Information Technologies, 25 (2019), 2481 - 2520.

[Peña-Ríos et al. 2012] Peña-Ríos, A., Callaghan, V., Gardner, M. and Alhaddad, M.J: "Remote mixed reality collaborative laboratory activities: Learning activities within the InterReality Portal."; Proc. IEEE/WIC/ACM International Conferences on Web Intelligence and Intelligent Agent Technology, 3 (2012), 362 - 366.

[Podwal 2016] Podwal, M.: "Google Earth VR - Bringing the whole wide world to virtual reality" (Google AR and VR Product Manager at Google Inc.). Retrieved May 8, 2018, from https://www.blog.google/products/google-vr/google-earth-vrbringing-whole-wide-world-virtual-reality/ (2016). 
[Pollard eet al. 2020] Pollard, K.A., Oiknine, A.H., Files, B.T., Sinatra, A.M., Patton, D., Ericson, M., Thomas, J. Khooshabeh, P.: "Level of immersion affects spatial learning in virtual environments: results of a three-condition within-subjects study with long intersession intervals"; Virtual Reality (2020), 1 - 14.

[Sánchez et al. 2000] Sánchez, Á., Barreiro, J. M., Maojo, V.: "Design of virtual reality systems for education: a cognitive approach"; Education and information technologies, 5, 4 (2000), $345-362$.

[Sebrechts et al. 2003] Sebrechts, M. M., Lathan, C., Clawson, D. M., Miller, M. S., Trepagnier, C.: "Transfer of training in virtual environments: Issues for human performance" (1 ed.). London: Lawrence Erlbaum Associates (2003).

[Shneiderman 2010] Shneiderman, B., Plaisant, C.: "Designing the user interface: strategies for effective human-computer interaction"; London: Pearson Education (2010).

[Song and Lee 2002] Song, K. S., Lee, W. Y.: "A virtual reality application for geometry classes. Journal of Computer Assisted Learning, 18, 2 (2002), 149 - 156.

[Statista 2020] Statista: Statista Research Department; "Virtual Reality (VR) - Statistics Facts"; Retrieved April 27, 2020, from https://www.statista.com/topics/2532/virtual-reality-vr/ (2020).

[Stuart and Thomas 1991] Stuart, R., Thomas, J.: "The Implications of Education in Cyberspace"; Multimedia Review, 2 (1991), 17 - 27.

[Timoney et al. 2018] Timoney, J., Faghih, B., Gibney, A., Korady, B., Young, G. W.: "Singing-Blocks: Considerations for a Virtual Reality Game to create chords and progressions"; Proc. Computer Simulation of Musical Creativity, Dublin (2018).

[University of Toronto 2019] University of Toronto: "Virtual Reality in the Classroom: Best Practices for Using Virtual Reality in Education"; Retrieved May 11, 2020, from https://guides.library.utoronto.ca/c.php?g=607624p=4494486 (2019).

[Vagg et al. 2016] Vagg, T., Plant, B. J., Ronan, N., Eustace, J., Tabirca, S.: "A Cystic Fibrosis Virtual Reality Gamified Experience for Medical Students"; Proc. Irish Conference on Game-Based Learning, Dublin (2016).

[Virvou and Katsionis 2008] Virvou, M., Katsionis, G.: "On the usability and likeability of virtual reality games for education: The case of VR-ENGAGE"; Computers Education, 50, 1 (2008), 154 - 178.

[Vora et al. 2002] Vora, J., Nair, S., Gramopadhye, A. K., Duchowski, A. T., Melloy, B. J., Kanki, B.: "Using virtual reality technology for aircraft visual inspection training: presence and comparison studies"; Applied Ergonomics, 33, 6 (2002), 559 -570 .

[Warburton 2009] Warburton, S.: "Second Life in higher education: Assessing the potential for and the barriers to deploying virtual worlds in learning and teaching"; British journal of educational technology, 40, 3 (2009), 414 - 426.

[Wohlgenannt et al. 2019] Wohlgenannt, I., Fromm, J., Stieglitz, S., Radianti, J., Majchrzak, T. A.: "Virtual Reality in Higher Education: Preliminary Results from a Design-Science-Research Project"; Proc. Information Systems Development: Information Systems Beyond 2020, Toulon (2019).

[Yoo et al. 2018] Yoo, M., Kim, J., Koo, Y., Song, J. H.: "A meta-analysis on the effects of VR, AR, MR-based learning in Korea"; The Journal of Educational Information and Media, 24, 3 (2018), 459 - 488.

[Young et al. 2017] Young, G. W., Murphy, D., Weeter, J.: "A Qualitative Analysis of Haptic Feedback in Music Focused Exercises"; Proc. New Interfaces for Musical Expression, Copenhagen (2017), 204 - 209.

[Youngblut 1998] Youngblut, C.: "Educational Uses of Virtual Reality Technology"; Report No. IDA-D-2128, Alexandria: Institute for Defense Analyses (1998).

[Zelle and Figura 2004] Zelle, J., Figura, C.: "Simple, low-cost stereographic: VR for everyone"; ACM SIGCSE Bulletin, 36, 1 (2004), 348 - 352. 